\title{
CORRELATION OF MORPHOLOGIC AND PRODUCTION TRAITS OF HONEY BEE COLONIES FROM SERBIA
}

\author{
G. Jevtić ${ }^{1}$, B. Andjelković ${ }^{1}$, Z. Lugić ${ }^{1}$, D. Djokić ${ }^{1}$, M. Mladenović ${ }^{2}$, N. \\ Nedić $^{2}$ \\ ${ }^{1}$ Institute for Forage Crops, Kruševac, Republic of Serbia \\ ${ }^{2}$ Faculty of Agriculture, Belgrade-Zemun, Republic of Serbia \\ Corresponding author: goran.jevtic@ikbks.com \\ Original scientific paper
}

Abstract: State of mutual or reciprocal relationship between traits is determined by the correlation coefficients. Correlation of morphological and production traits was determined in two generations of honey bee colonies. In the first generation, mother queen bees were studied, and in the second generation, their offspring. The study of one generation lasted two years. Morphological traits were determined in the beginning of the study and production traits were determined in the spring and autumn inspections of every year. In the first generation, the positive and mostly high correlation between morphological traits, and honey yield and hygienic behaviour was determined. Correlation of morphological traits, colony strength and food supplies per colony ranged from highly positive to slightly negative values. It was found that there was a high correlation between some production traits (colony strength in the spring and autumn inspections and honey yield per colony). In the analysis of offspring (second generation), most traits that were positively correlated in mother generation now, also, showed a similar relationship. The only difference, in contrast to mother generation, was that, for most traits, the intensity of interaction increased.

Key words: honey bee, correlation coefficient, morphologicl traits, production traits

\section{Introduction}

Correlation is interrelationship between the occurrences presented by different values of the two variables. This connection means that the value of variables can be predicted with a certain probability based on knowledge of the values of other variables. Correlation coefficients measure expression of the relationship between two variables in the units independently of the specific units 
of measure of variables. Determining the correlation relationship between different traits is especially important in honey bee breeding programs. Particular attention to correlation relationships between different traits in honey bees and was given by Билаш and Кривцов (1991). In sampling of the colonies for further selection, indirect selection methods based on the correlation coefficients are placed just behind the method of large-scale phenotypic selection and methods of genotypic individual selection. They claim that this method is based on the correlative variability, meaning that change in one of traits triggers changes in other traits which are in the correlation relationship with previous trait.

Above mentioned authors claim that the honey yield is in positive correlation with tongue length $(r=0.22$ to 0.72$)$, front wing length $(r=0.69)$, front wing width $(r=0.31)$, third tergit length $(r=0.63)$ and the cubital index $(r=0.32)$. As for the other traits of the honey bee colony, productivity is the most correlated with the number of eggs laid by honey bee queen per day 36 days from the beginning of foraging $(r=0.73)$ and intensity of bee flights $(r=0.62)$. They state that the tongue length is negatively correlated with fertility of queen bee and resistance to Nosema. Mladenović and Radoš (2010) have established the existence of a positive correlation between the colony strength and the amount of honey and pollen in the colony. The highest level of interaction is achieved between the number of bees and the amount pollen in the colony $(r=0.48)$, while the lowest recorded interaction is between the amount of pollen and the amount of honey $(\mathrm{r}=0.13)$. The honey yield is correlated with the lifespan of worker bees (Milne, 1980), one-day intake of honey (Szabo, 1981), brood area (Georgiev and Plavša, 2005), meteorological factors and the capacity of the honey flora (Mladenovic, 2006) and other factors.

The aim of this study was to determine the correlation coefficients of morphological traits (tongue length, length and width of the wing and basitarsus) and production characteristics of honey bee (colony strength and food supply) as determined in the autumn and spring examination with a total yield of honey per company and the expressed hygienic behaviour.

\section{Materials and Methods}

In this experiment, 18 colonies were studied. Colonies were sampled from different regions of Serbia (Rasina, Pester, Morava, Banat, Timok and Kopaonik). Morphological traits were determined using binocular, brand "Olympus", with a magnification $25 \mathrm{x}$, which has a measuring eyepiece which was used for the measurement of honey bee body parts. During the spring examination, 50 bees were taken from each colony and suffocated, then their body parts were separated (head, wing and leg), placed on the slide and measured using binocular. 
The amount of bees, brood area, honey and pollen in the colony were estimated visually during the spring (the last decade of March) and autumn examinations (the first decade of September). These traits are expressed in taken size per frame $(1 / 10)$ or in percentage. Honey yield was determined as the total honey subtracted from the colony with the honey left for the winter. The amount of honey left for the winter is determined by the area of caped honey on $1 \mathrm{dm} 2$ calculated as $0.25 \mathrm{~kg}$ and $1 \mathrm{dm} 2$ uncapped as $0.15 \mathrm{~kg}$ of honey.

Hygienic behaviour was determined using pin killed method.

For morphological traits (tongue length, length and width of tarsus), production characteristics and quantity of collected honey, correlation coefficients were calculated according to formulas by Hadživuković (1991). Correlation coefficients were calculated separately for the parent colonies and for offspring colonies. The correlation coefficients were calculated by the following formula:

$$
r=\frac{\sum_{i=1}^{n}(X i-\bar{X})(Y i-\bar{Y})}{\sum_{i=1}^{n}(X i-\bar{X})^{2} \sum_{i=1}^{n}(Y i-\bar{Y})^{2}}
$$

Following scale was used for the strength of correlation:

0.00 to 0.30 - weak correlation;

0.31 to 0.60 - medium strong correlation;

0.61 to 0.90 - strong correlation;

Standard errors of correlation coefficients were calculated by the formula (Hadživuković, 1991):

$$
S_{r}=\frac{\sqrt{1-r^{2}}}{\sqrt{n-2}}
$$

Testing of the significance of correlation coefficients was performed by ttest using the following formula and table by Snedecor (Hadživuković, 1991):

$$
t=\frac{r}{S r}
$$

for $\mathrm{n}-2$ freedom degrees from the table of $\mathrm{t}$-distribution, where $\mathrm{n}$ is the number of colonies in the experiment. 


\section{Results and Discussion}

Correlation coefficients of the basic traits in parents. In has been established that in parent colonies there is a strong correlation between the morphological traits and honey yield and between morphological traits themselves, except for tarsal index which is negatively correlated with all morphological traits (Table 1). The amount of bees in the spring inspection was in strong correlation with brood area and pollen in both, spring and autumn, inspections, the honey yield and the number of foragers before and after noon. The amount of bees in the spring was in the medium-high correlation with the amount of bees in the autumn inspection. The amount of bees in the spring inspection was in strong correlation with brood area in the autumn and the honey yield. Brood area in the autumn was in strong correlation with the honey area in the spring of and honey yield, and in medium strong correlation with the honey area and pollen in autumn. Honey area in the autumn was in strong correlation with the honey yield per colony. Other traits were mainly in the medium strong correlative relationships. In parent colonies, honey yield was in strong and medium strong correlative relationships with most of the observed traits. From the observed traits, amount of honey in spring and autumn inspections showed the least correlation with other observed traits. Manifested hygienic behavior was determined in 24 and 48 hours after damaging the cells, and it showed a strong correlation with the wing width $(\mathrm{r}=$ 0.61 and $r=0.53)$ and medium-strong correlation with the tongue length $(r=0.38)$. It was determined that colonies that exhibit hygienic behavior in the first 24 hours after the damage, also do it after 48 hours $(\mathrm{r}=0.72)$. Hygienic behavior was in a weak or negative correlation with any other production traits 
Table 1 The correlation coefficients for the productive traits of the maternal colonies

\begin{tabular}{|c|c|c|c|c|c|c|c|c|c|c|c|c|c|c|c|}
\hline 号总 & $\mid \begin{array}{l}\infty \\
m \\
0 \\
0\end{array}$ & $\begin{array}{l}m \\
0 \\
0 \\
1\end{array}$ & $\begin{array}{l}-1 \\
0\end{array}$ & $\begin{array}{l}0 \\
\text { ㄴ. } \\
0\end{array}$ & సิ & $\begin{array}{l}2 \\
\overrightarrow{0} \\
1\end{array}$ & $\begin{array}{l}m \\
0\end{array}$ & s. & 요 & $\begin{array}{l}0 \\
3 \\
0 \\
1\end{array}$ & $\begin{array}{l}8 \\
0 \\
1\end{array}$ & $\begin{array}{l}m \\
3 \\
0 \\
1\end{array}$ & $\begin{array}{l}\overrightarrow{7} \\
\text { ? } \\
\text { ? }\end{array}$ & $\begin{array}{l}J \\
0 \\
0\end{array}$ & $\frac{0}{2}$ \\
\hline 空空 & $\mid \begin{array}{l}\infty \\
m \\
0\end{array}$ & $\begin{array}{l}8 \\
0 \\
0 \\
1\end{array}$ & ली & ?ీ & $\frac{\infty}{0}$ & $\begin{array}{l}2 \\
\text { ?ี } \\
0 \\
1\end{array}$ & $\begin{array}{l}n \\
8 \\
0\end{array}$ & $\frac{n}{0}$ & ปे & $\begin{array}{l}\infty \\
0 \\
0 \\
1\end{array}$ & $\begin{array}{l}0 \\
\text { ஸी } \\
0\end{array}$ & $\begin{array}{l}8 \\
8 \\
0\end{array}$ & $\frac{m}{0}$ & ○̊ & \\
\hline рцәฺ̆ Кәшон & $\begin{array}{l}0 \\
\stackrel{0}{0} \\
0\end{array}$ & $\begin{array}{l}0 \\
0 \\
0\end{array}$ & $\stackrel{m}{0}$ & $\stackrel{0}{+}$ & $\vec{m}$ & $\frac{n}{0}$ & $\begin{array}{l}\overrightarrow{0} \\
0 \\
0\end{array}$ & $\frac{m}{0}$ & : & तે & $\begin{array}{l}8 \\
8 \\
0\end{array}$ & $\vec{n}$ & $\frac{0}{0}$ & & \\
\hline 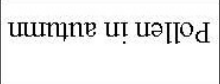 & $\underset{0}{\infty}$ & $\begin{array}{l}\circ \\
0 \\
\end{array}$ & \begin{tabular}{l}
$\infty$ \\
\multirow{1}{0}{} \\
0
\end{tabular} & $\Rightarrow$ & $\frac{1}{0}$ & $\begin{array}{l}\infty \\
8 \\
0 \\
0\end{array}$ & $\begin{array}{l}8 \\
8 \\
0\end{array}$ & हn & 8 & $\frac{n}{0}$ & $\begin{array}{l}\circ \\
0 \\
0\end{array}$ & $\begin{array}{l}8 \\
\infty \\
0\end{array}$ & & & \\
\hline 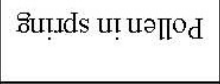 & $\begin{array}{l}n \\
0 \\
i\end{array}$ & तี & $\begin{array}{l}\text { ?a } \\
\text { î }\end{array}$ & $\frac{0}{0}$ & 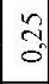 & $\begin{array}{l}0 \\
0 \\
0 \\
1\end{array}$ & $\begin{array}{l}8 \\
0 \\
0\end{array}$ & $\begin{array}{l}m \\
0\end{array}$ & $\begin{array}{l}0 \\
0 \\
0\end{array}$ & ปี & $\begin{array}{l}7 \\
7 \\
0\end{array}$ & & & & \\
\hline 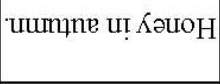 & $\overrightarrow{0}$ & $\begin{array}{l}\text { ปี } \\
\text { ᄋ }\end{array}$ & $\begin{array}{l}5 \\
8 \\
0\end{array}$ & กี & 今 & 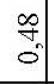 & $\begin{array}{l}0 \\
\text { की } \\
0\end{array}$ & ชิ| & के. & in & & & & & \\
\hline ઠిuழ̣ds u! Кәu०H & $\begin{array}{l}\hat{N} \\
0 \\
i\end{array}$ & $\frac{m}{0}$ & 요 & $\begin{array}{l}0 \\
0 \\
0\end{array}$ & $\begin{array}{l}m \\
0 \\
0\end{array}$ & $\begin{array}{l}0 \\
\text { ले } \\
0\end{array}$ & $\begin{array}{l}-7 \\
0 \\
0\end{array}$ & $\begin{array}{l} \pm \\
\\
i\end{array}$ & $\begin{array}{l}\delta \\
\text { o } \\
i\end{array}$ & & & & & & \\
\hline $\begin{array}{c}\text { uumßne } \\
\text { u!̣ вә.上 pooIg }\end{array}$ & $\begin{array}{l}\mathcal{O} \\
\delta \\
0\end{array}$ & 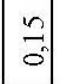 & $\begin{array}{l}2 \\
8 \\
0\end{array}$ & ले & $\underset{\ddagger}{\sharp}$ & $\begin{array}{l}0 \\
0 \\
0 \\
1\end{array}$ & รั) & $\mid \begin{array}{l}\infty \\
\infty \\
0 \\
0\end{array}$ & & & & & & & \\
\hline 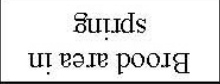 & $\begin{array}{l}5 \\
0 \\
0 \\
\end{array}$ & $\begin{array}{l}\text { ?. } \\
\text { ? } \\
\end{array}$ & $\begin{array}{l}0 \\
0 \\
0\end{array}$ & $\begin{array}{l}5 \\
0 \\
\end{array}$ & ? & $\begin{array}{l}8 \\
8 \\
0\end{array}$ & $\begin{array}{l}\infty \\
\infty \\
0\end{array}$ & n. & & & & & & & \\
\hline $\begin{array}{c}\text { uuminne } \\
\text { u! səəq Jo łunoury }\end{array}$ & $\stackrel{m}{0}$ & $\begin{array}{l}5 \\
0 \\
0\end{array}$ & $\begin{array}{l}2 \\
8 \\
0\end{array}$ & $\begin{array}{l}8 \\
8 \\
0\end{array}$ & $\stackrel{m}{f}$ & $\left|\begin{array}{l}0 \\
0 \\
0 \\
1\end{array}\right|$ & $\vec{n}$ & & & & & & & & \\
\hline $\begin{array}{c}\text { ôutuds } \\
\text { u! səəq Jo ұunoury }\end{array}$ & $\begin{array}{l}7 \\
0 \\
0\end{array}$ & $\begin{array}{c}9 \\
0 \\
0\end{array}$ & $\begin{array}{l}8 \\
8 \\
0 \\
1\end{array}$ & ची & 8 & $\begin{array}{l}0 \\
0 \\
1\end{array}$ & & & & & & & & & \\
\hline xәрu! [eS.IP $L$ & $\begin{array}{l}\text { กิ } \\
\text { ? } \\
1\end{array}$ & $\begin{array}{l}\infty \\
0 \\
0 \\
1\end{array}$ & ?. & กี. & ?ֶ. & & & & & & & & & & \\
\hline પIPIM SnSIEI!SE & $\begin{array}{l}0 \\
+ \\
0 \\
0\end{array}$ & 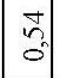 & 이 & $\begin{array}{l}2 \\
0 \\
0\end{array}$ & & & & & & & & & & & \\
\hline 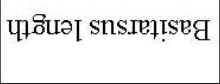 & ñ & 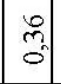 & की & & & & & & & & & & & & \\
\hline чрртм о̊и! $M$ & $\hat{n}$ & $\begin{array}{l}\text { הู } \\
0 \\
\end{array}$ & & & & & & & & & & & & & \\
\hline 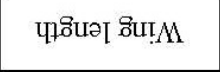 & $=$ & & & & & & & & & & & & & & \\
\hline & 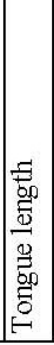 & 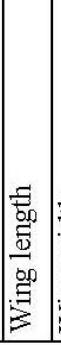 & 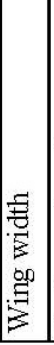 & 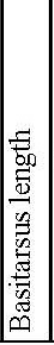 & 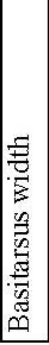 & 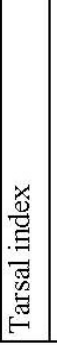 & 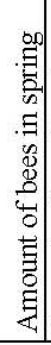 & 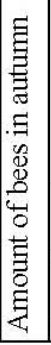 & 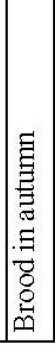 & 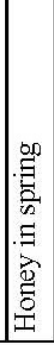 & 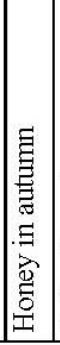 & 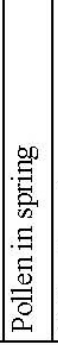 & 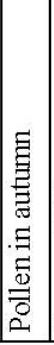 & 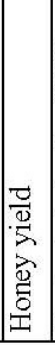 & 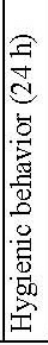 \\
\hline
\end{tabular}


Correlation coefficients of basic traits in offspring. When testing offspring (queen bee daughters) in the third and fourth year, most traits which were positively correlated in their parents also showed positive correlation. The only difference when compared to the parents is that for most correlated traits, the intensity of mutual influence (correlation coefficient) was increased (Table 2). Morphological traits were in the medium heavy and strong correlation with each other, except tarsal index, which had a negative correlated to the other morphological traits. Colony strength (the amount of bees and the brood area) was in medium-high and a strong correlation with the honey yield per colony. The highest correlation $(r=0.91)$ was determined for the amount of bees and the brood area in the spring. Food supply (quantity of honey and pollen) showed minimum correlation with the majority of traits (low or negative correlation). Hygienic behavior of colonies also had very low correlation with majority of traits (mostly negative). The exception was the count after 24 hours and after 48 hours where there was a strong correlation $(\mathrm{r}=0.72)$, which is quite understandable. Hygienic behavior and wing width had medium-strong or strong correlation $(\mathrm{r}=0.58$ and $\mathrm{r}=$ 0.64) which is very interesting. Bilaš and Krivcov (1991) state that the tongue length in the Caucasian race of honey bee was positively correlated with the honey yield, however, the same research found that the tongue length was negatively correlated with fertility of queen bees and resistance to the Nosema. In addition to the tongue length foraging was highly correlated to the length of the front wing, the third tergit length, maximum number of eggs laid by queen bee, flight intensity, etc. In this source several authors were also cited (Gold, 1937; Miller, 1939; Bessonet, 1945; Bichtler, 1951; Hunkeller, 1953) who claim that the honey yield is not in correlation with morphological traits of honey bees. The two-year research by Plavša and Georgiev (2005), it was proved that there was a positive correlation between the amount of bees and brood and the productivity of colonies or the honey yield. Lebedev (2001) announces that the input of pollen was in high correlative dependence $(\mathrm{r}=0.82)$ to the colony strength and the amount of brood per colony. Georgiev et al. (2003) found that the correlation coefficient between the amount of brood and the amount of pollen in the spring was very high while in the fall it was much lower. 
Table 2 The correlation coefficients for the productive traits of the offspring colonies

\begin{tabular}{|c|c|c|c|c|c|c|c|c|c|c|c|c|c|c|c|c|}
\hline 运. & ले & 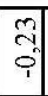 & $\begin{array}{l}\vec{b} \\
0\end{array}$ & 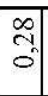 & $\begin{array}{l}\text { Na } \\
\text { o }\end{array}$ & $\begin{array}{l}0 \\
\stackrel{0}{1} \\
0\end{array}$ & $\begin{array}{l}\text { m. } \\
0\end{array}$ & $\begin{array}{l}\text { ภे } \\
0\end{array}$ & $\begin{array}{l}\text { ली. } \\
\text { ᄋ }\end{array}$ & $\begin{array}{l}1 \\
0 \\
0\end{array}$ & $\begin{array}{l}0 \\
0 \\
0\end{array}$ & $\begin{array}{l}m \\
\text { J } \\
0 \\
1\end{array}$ & $\vec{z}$ & \begin{tabular}{|l|} 
\\
\\
0
\end{tabular} & \begin{tabular}{|l|}
8 \\
0 \\
0
\end{tabular} & $\begin{array}{l}m \\
\infty \\
0\end{array}$ \\
\hline 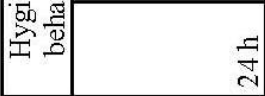 & $\begin{array}{l}0 \\
\text { กे. } \\
0\end{array}$ & $\begin{array}{l}8 \\
0 \\
0 \\
1\end{array}$ & $\begin{array}{l}\infty \\
2 \\
0 \\
0\end{array}$ & $\begin{array}{l}- \\
0 \\
0\end{array}$ & $\begin{array}{l}\vec{\lambda} \\
0 \\
0\end{array}$ & $\begin{array}{l}\text { In } \\
0 \\
0\end{array}$ & $\overrightarrow{0}$ & $\begin{array}{l}0 \\
\text { 연 }\end{array}$ & की & 응 & $=$ & $\begin{array}{l}0 \\
0 \\
0 \\
1\end{array}$ & $\begin{array}{l}m \\
m \\
0\end{array}$ & \begin{tabular}{|c|}
$\infty$ \\
$n$ \\
0 \\
\end{tabular} & $\begin{array}{l}5 \\
0 \\
1\end{array}$ & \\
\hline рјәฺุ КәиоН & $\begin{array}{l}1 \\
0 \\
0\end{array}$ & 영 & $\begin{array}{l}\text { 이 } \\
\text { of }\end{array}$ & $\begin{array}{l}\text { of } \\
\text { of }\end{array}$ & $\begin{array}{l}\infty \\
m \\
0 \\
0\end{array}$ & $\begin{array}{l}\exists \\
\overrightarrow{0}\end{array}$ & $\begin{array}{l}-1 \\
0 \\
0\end{array}$ & 종 & है & के & \begin{tabular}{l|}
0 \\
-1 \\
0
\end{tabular} & ?] & $\begin{array}{l}\infty \\
\overrightarrow{0}\end{array}$ & $\begin{array}{l}\text { ते } \\
\text { o }\end{array}$ & & \\
\hline 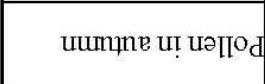 & $\begin{array}{l}2 \\
0 \\
1 \\
\end{array}$ & 8 & $\begin{array}{l}\hat{2} \\
\hat{q} \\
\hat{i}\end{array}$ & शे & 馬 & $\begin{array}{l}0 \\
8 \\
0 \\
0\end{array}$ & $\begin{array}{l}\text { गे } \\
\text { ठ }\end{array}$ & $\begin{array}{l}\text { 영 } \\
0\end{array}$ & ले & $\begin{array}{l}9 \\
0 \\
0\end{array}$ & $\begin{array}{l}0 \\
0 \\
0\end{array}$ & $\begin{array}{l}7 \\
0 \\
0\end{array}$ & $=$ & & & \\
\hline ôuụds u!̣ uəI[ం d & \begin{tabular}{l|}
- \\
0 \\
\end{tabular} & $\begin{array}{l}\text { गे } \\
\text { of }\end{array}$ & 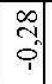 & $\frac{1}{0}$ & $\begin{array}{l}\mid+1 \\
\text { m. } \\
0\end{array}$ & $\begin{array}{c}\text { I } \\
0 \\
i\end{array}$ & $\begin{array}{l}0 \\
\text { N } \\
0\end{array}$ & $\begin{array}{l}\overrightarrow{0} \\
0 \\
0\end{array}$ & ले & $\frac{1}{0}$ & $\begin{array}{l}0 \\
\text { } \\
0 \\
1\end{array}$ & $\begin{array}{l}2 \\
\text { an } \\
0 \\
0\end{array}$ & & & & \\
\hline 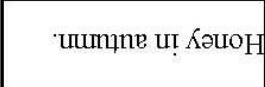 & \begin{tabular}{l|l|} 
& 0 \\
0 \\
0 \\
1
\end{tabular} & $\begin{array}{l}\text { क् } \\
\text { ô }\end{array}$ & $\begin{array}{l}0 \\
0 \\
0 \\
1\end{array}$ & ?ี & $\begin{array}{l}\text { 今े } \\
\text { ô }\end{array}$ & $\begin{array}{l}\text { तี } \\
\text { की }\end{array}$ & $\begin{array}{l}\overrightarrow{2} \\
\text { î }\end{array}$ & $\begin{array}{l}\infty \\
\text { त } \\
0\end{array}$ & $\begin{array}{l}\infty \\
\stackrel{0}{0} \\
0\end{array}$ & $\begin{array}{l}\text { जิ } \\
\text { o }\end{array}$ & $\begin{array}{l}0 \\
\text { กิ }\end{array}$ & & & & & \\
\hline ôụıds uा КəuоH & न & 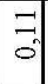 & $\begin{array}{l}3 \\
\text { తn } \\
0\end{array}$ & $\begin{array}{l}\vec{Z} \\
\bar{i}\end{array}$ & $\begin{array}{l}3 \\
0 \\
0\end{array}$ & $\begin{array}{l}7 \\
\text { ஸे } \\
0\end{array}$ & $\begin{array}{l}3 \\
0 \\
0\end{array}$ & वे & $\begin{array}{l}\text { गे } \\
0\end{array}$ & 고 & & & & & & \\
\hline 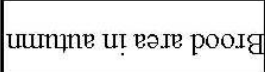 & लै & $\begin{array}{l}0 \\
8 \\
0\end{array}$ & ने & $\begin{array}{l}\stackrel{y}{\checkmark} \\
0 \\
0\end{array}$ & ?ָ & $\begin{array}{l}0 \\
8 \\
0 \\
1\end{array}$ & $\begin{array}{l}n \\
\text { ح̂. } \\
0\end{array}$ & $\begin{array}{l}\text { ปे } \\
\text { of }\end{array}$ & 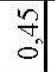 & & & & & & & \\
\hline ôuụds u!̣ pəIt pooıg & $\begin{array}{l}\text { च } \\
\text { i }\end{array}$ & $\begin{array}{l}\text { त्र } \\
\text { î }\end{array}$ & $\begin{array}{l}m \\
0 \\
0\end{array}$ & $\frac{m}{0}$ & $\begin{array}{l}0 \\
0 \\
0 \\
i\end{array}$ & $\begin{array}{l}\delta \\
0 \\
0\end{array}$ & $\begin{array}{l}\vec{\sigma} \\
0\end{array}$ & जू. & & & & & & & & \\
\hline $\begin{array}{r}\text { uummn } \\
\text { u! səəq Jo łunoury }\end{array}$ & $\begin{array}{l}\infty \\
0 \\
0\end{array}$ & $\frac{9}{0}$ & $\frac{9}{0}$ & $\begin{array}{l}0 \\
6 \\
0\end{array}$ & 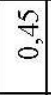 & $\begin{array}{l}\text { so } \\
0 \\
0\end{array}$ & ת & & & & & & & & & \\
\hline $\begin{array}{l}\text { out..ds } \\
\text { u! səaq jo ұunoury }\end{array}$ & ते & $\begin{array}{l}9 \\
? \\
1\end{array}$ & $\begin{array}{l}0 \\
0 \\
0 \\
1\end{array}$ & $\begin{array}{l}0 \\
0 \\
0\end{array}$ & $=$ & $\begin{array}{l}8 \\
8 \\
0\end{array}$ & & & & & & & & & & \\
\hline хәрu! ןеs.t. & $\begin{array}{l}\text { ले } \\
\text { की }\end{array}$ & $\begin{array}{l}7 \\
0 \\
0\end{array}$ & $\begin{array}{l}\text { ले } \\
\text { of }\end{array}$ & 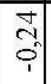 & 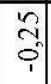 & & & & & & & & & & & \\
\hline ЧРP!M SnSIRł!SEG & गे & $\begin{array}{l}-1 \\
0 \\
0\end{array}$ & $\begin{array}{l}\text { ते } \\
\text { of }\end{array}$ & $\overrightarrow{0}$ & & & & & & & & & & & & \\
\hline 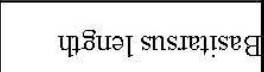 & \begin{tabular}{|l|} 
\\
6 \\
0 \\
0
\end{tabular} & $\begin{array}{l}\infty \\
\text { s } \\
0\end{array}$ & $\begin{array}{l}\infty \\
m \\
0 \\
0\end{array}$ & & & & & & & & & & & & & \\
\hline чıрเм о̊น!M & $\begin{array}{l}n \\
0 \\
0\end{array}$ & $\begin{array}{l}n \\
\text { m. } \\
0\end{array}$ & & & & & & & & & & & & & & \\
\hline 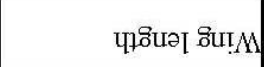 & - & & & & & & & & & & & & & & & \\
\hline & 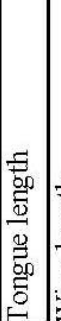 & 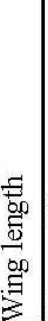 & 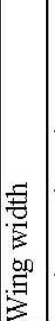 & 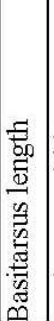 & 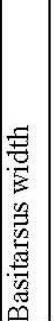 & 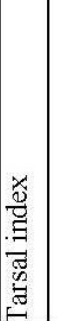 & 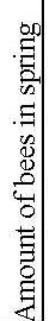 & 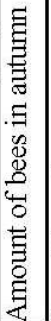 & 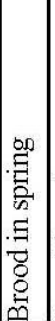 & 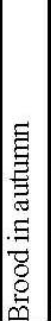 & 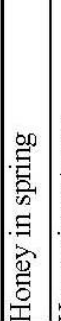 & 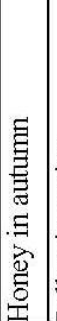 & 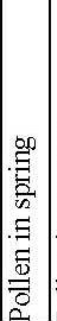 & 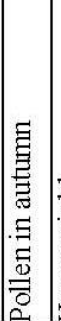 & 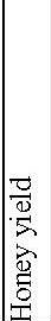 & 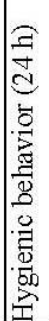 \\
\hline
\end{tabular}




\title{
Conclusion
}

During the four-year study of two generations of honey bee colonies it was determined that some morphological and some production traits have a major impact on the overall productivity of colony or the honey yield of.

Most morphological traits (except tarsal index) showed a medium strong and strong correlation to each other and to the productivity of colony.

Colony strength (the amount of bees and the brood area) in both generations exhibited strong and medium strong correlation with the honey yield. Weaker correlation was determined between food supply (amount of honey and pollen) with the honey yield of honey, and this is particularly evident in the second generation.

Expression of hygienic behaviour in the colony had a low correlation to the majority of traits. Only the wing width $(\mathrm{r}=0.58$ and $\mathrm{r}=0.64)$ and tongue length $(\mathrm{r}$ $=0.36$ and $r=0.39$ ) had showed strong or medium-strong correlation with this trait

\section{Korelacija morfoloških i proizvodnih osobina društava medonosne pčele sa područja Srbije}

\author{
G. Jevtić, B. Andjelković, Z. Lugić, D. Djokić, M. Mladenović, N. Nedić
}

\section{Rezime}

Stanje uzajamne ili recipročne povezanosti jedne osobine od druge utvrđuje se na osnovu koeficijenta korelacije. Korelacija morfoloških i proizvodnih osobina utvrđivana je kroz dve generacije društava medonosne pčele. U prvoj generaciji praćene su majke matice, a u drugoj njihovo potomstvo. Ispitivanje po jednoj generaciji trajalo je dve godine. Morfološke osobine su utvrđene na početku praćenja generacija, a proizvodne na prolećnom i jesenjem pregledu svake godine.

U prvoj generaciji utvrđena je pozitivna i mahom visoka korelacija između morfoloških osobina, prinosa meda i higijenskog ponašanja. Stepen korelacije morfoloških osobina, snage društva i zaliha hrane po društvu bio je od visoko pozitivne do slabo negativne vrednosti. Ustanovljeno je da postoji i visok stepen korelacije između pojedinih proizvodnih osobina (snaga društava na prolećnom i jesenjem pregledu i prinosa meda po društvu).

Prilikom testiranja potomstva (II generacija) većina osobina koje su bile u pozitivnoj korelaciji kod roditelja i sada su pokazivala uzajamni odnos. Jedina razlika $\mathrm{u}$ odnosu na roditelje je $\mathrm{u}$ tome da se, za većinu osobina, intezitet međusobnog uticaja povećao. 


\section{References}

БИЛАШ Г.Д., КРИВЦОВ Н.И. (1991): Селекция пчел. Изд. “Агропромиздат” Москва, 304.

GEORGIJEV A., MLADENOVIĆ M., NEDIĆ N. (2003): Experimental calculation of the correlation between the cell surface and the intake of nectar and pollen in bee colonies. XXXVIIIth Apimondia International Apicultural Congress. August 24-29. Ljubljana, Slovenia, 760.

GEORGIJEV A., PLAVŠA N. (2005): Korelacija između površine legla i pčela na produktivnost pčelinjih društava. XIII Naučno savetovanje sa međunarodnim učešćem, 12-13. februar, Poljoprivredni fakultet, Zemun, Zbornik radova, 107-112. HADŽIVUKOVIĆ S. (1991): Statistički metodi. Drugo prošireno izdanje, Izdavač Poloprivredni fakultet, Institut za ekonomiku poljoprivrede i sociologiju sela, Univerzitet u Novom Sadu - Novi Sad.

ЛЕБЕДЕВ В.И. (2001): Биологический потенциал пчелиној семи по сбору и загатовке перги. Материял координационог совещания и конференции, Масква, 60-63.

MILNE P. (1980): Laboratory measurement of honey production in honey bee. Longevity or length of life of caged workers. Jour. of Apic. Res., 19, 172-175.

MLADENOVIĆ M. (2006): Medna produktivnost nekih selekcijskih linija matica u Srbiji. XIV Naučno savetovanje sa međunarodnim učešćem, 11-12. februar, Poljoprivredni fakultet Zemun, Zbornik radova, 7-13.

MLADENOVIĆ M., RADOŠ R. (2010): Correlation between the strength of colony, the honey area and pollen area of the observed lines of yellow honey bbe in Vojvodina. Biotechnol. \& Biotechnol. EQ. 24/2010/SE Special edition. Second Balkan Conference on Biology 21-23 May 2010, Plovdiv, 423-426.

SZABO T.I. (1982): Correlations between colony traits in the honeybee. American Bee Journal, 112, 611-616. 